\title{
Digital Annotations: a Formal Model and its Applications
}

\author{
Nicola Ferro \\ Department of Information Engineering - University of Padua \\ Via Gradenigo, 6/b - 35131 Padua - Italy \\ ferro@dei.unipd.it
}

\begin{abstract}
We discuss various issues and viewpoints concerning the annotation of digital contents, such as textual documents, images, and multimedia documents in general. The discussion shows how fragmentary the picture is about the annotation and how its definition changes according to the different applicative contexts in which it is used. Therefore, we propose a formal model of the annotation which provides us with a unified and integrated picture on the annotation which takes into account the different viewpoints and uses of it. Finally, we present various possible application areas of the proposed formal model and we introduce the next steps we can undertake by using it as a starting point.
\end{abstract}

Key words: annotation, digital content, foundations, hypertext, information management system

\section{Introduction}

The possibility of enriching and personalizing digital contents by adding and sharing annotations has attracted many researchers, who have looked at this opportunity from many different perspectives and with a number of purposes in mind.

Almost everybody is familiar with annotations and has his own intuitive idea about what they are, drawn from personal experience and the habit of dealing with some kind of annotation in every day life, which ranges from jottings for the shopping to taking notes during a lecture or even adding a commentary to a text. This intuitiveness makes annotations especially appealing for both researchers and users: the former propose annotations as an easy understandable way of performing user tasks, while the latter feel annotations to be a familiar tool for carrying out their own tasks. Therefore, annotations have been adopted in a variety of different contexts, such as content enrichment, data curation, collaborative and learning applications, and social networks, as well as in various information management systems, such as the Web (semantic and not), digital libraries, and databases. Surprisingly, 
all these approaches have not led us to deep knowledge and comprehension of the annotation but, on the contrary, the picture about the annotation of digital contents is still quite fragmentary.

Even though this concept is so familiar, it turns out to be especially elusive when it comes to explicitly and formally defining it, mainly because it is a far more complex and multifaceted concept than one might imagine at a first glance. Indeed, we usually derive what an annotation is from the particular task to which it is applied, rather than investigating the annotation by itself in order to understand its features and how to use it. To cite a few examples, if we deal with the Semantic Web, annotations are considered as metadata [43,70]; in collaborative applications annotations are a seen as a discourse [34] and might be considered even like e-mails [35]; in the field of digital libraries annotations are treated as an additional content $[2,8]$; when we talk about scientific databases, annotations represent both provenance information about the managed data and a way of curating the database itself $[26,28]$; in the case of data minining and data visualization, annotations are seen as a means for recording the history of user explorations in visualization environments [40]; finally, in social networks and collaborative tagging, annotations are tags or keywords on different kinds of digital content, e.g. photos or bookmarks [37]. It can be noted as these different notions of annotation partially overlap or share commonalities.

This flourishing of different viewpoints about the annotation, which are often considered as separated, reveals a lack of a clear comprehension of what an annotation is, prevents us from exploiting synergies and complementarities among the different approaches, and makes it more difficult to determine what the differences between annotations and other concepts are, and what the advantages or disadvantages of using annotations are, even when they seem so similar to other concepts.

Moreover, we should consider that the tools we adopt influence the way we work, may change the way we approach a problem, and may impact on our findings. Thus, if we are not able to define annotations and determine their features by themselves, we will not be able to understand whether and when annotations are the tool of choice for a given task, whether and when we can perform a task by using annotations better than by using other approaches, or what we lose or gain when we introduce the use of annotations with respect to other approaches to the same problem.

Therefore, we discuss the different perspectives regarding annotations in order to gather some key features about them. These key features can help us to better distinguish between the different uses of annotations and to understand the case at hand when dealing with annotations. Furthermore, they can be used as a support if we need to make design choices for developing an annotation management system. Finally, we use conducted analyses for proposing a comprehensive and unifying formal model of annotations on digital contents, which only recently has been introduced in the literature $[13,33]$. 
The proposed formal model intends to formalize the main concepts concerning annotations and define the relationships among annotations and annotated information resources. In addition, the formal model constitutes the necessary groundwork to be able to design and formalize search algorithms and to express query languages which take annotations into account, such as those proposed in $[11,12,35,38]$. Finally, the formal model provides us with the basis for designing and developing an annotation service which can be easily integrated into a wider Information Management System (IMS). Indeed, a clear definition of the concepts related to the annotation allows us to separate the functionalities needed to manage, access, and search annotations, which constitute the core of an annotation service, from the functionalities needed to integrate such an annotation service into IMSs.

The paper is organized as follows: Sect. 2 provides an overview of the different viewpoints about annotations; Sect. 3 provides an introduction to the proposed formal model and discusses different applications of it; Sect. 4 explains and formalizes the various concepts of the formal model; finally, Sect. 5 draws some conclusions and wraps up the discussion.

\section{Viewpoints on Annotations}

This section aims at giving readers a sample of the different viewpoints about annotations so that they can figure out the range of issues involved with annotations. Indeed, when we talk about annotations, we deal with a concept that has been stratified over a long period of time in our culture and comprises pre-existing knowledge from our cultural heritage. On the other hand, analysing the present use of annotations helps us to understand the current trends in developing annotation management systems as well as comprehend user requirements and expectations.

Therefore, we will discuss the annotation from three perspectives: the user perspective, as it is gained from user studies; the historical perspective on annotations, which provides us with additional information that users may not be able to point out because they often overlook what they have naturally absorbed from their cultural heritage or studies; finally, the perspective of the current annotation systems, which differently model annotations according to the tasks they aim at supporting. For each viewpoint, we will provide some relevant examples and related discussion.

Please note that this section does not intend to be a fully exhaustive survey on annotations, for which the interested reader can refer to $[5,13,31,43,55$, $58,60]$.

\subsection{User Viewpoint}

[51] studied personal annotative practices of American college students to point out the form the annotations have in the textbooks and the function of 
the annotations, which is derived from their form. [51, pp. 237-238] discovered that:

First, annotations are procedural signals, cluing in the student to where an assignment starts, what material is important (and as we will see, unimportant), and what material might require a second (or successive readings). Second, annotations are placemarks; they hold the quotes that are being reserved for the paper that the student will write at the end of the term, the chemical reactions and term definitions the student must memorize for the final, the theorem that is key to the proof in the homework assignment. Third, they are an in situ way of working problems. Fourth, annotations record interpretive activity, either from another reader (e.g. a professors explanation), or as the result of careful reading (the student has interpreted it him or herself). Fifth, and most elusively, these markings act as a visible trace of a reader's attention, a focus on the passing words, and a marker of all that has already been read (as if these words are now possessed). Finally, the markings may just be incidental, reflecting the material circumstance of reading.

[52] carries on her research work and categorizes annotations along several dimensions which reflect the form annotations may take on: formal versus informal annotations, explicit versus tacit annotations, annotations as writing versus annotations as reading, hyperextensive versus extensive versus intensive annotations, permanent versus transient annotations, published versus private annotations. Finally, [53], [54], and [65] investigate the relationship between private, shared and public annotations and how they can be exploited to find useful passages in the text.

Recently, [46] conducted a study on the impact of annotations in improving the learning achievements of students. A four-month experimentation was performed, where the learning achievements of student who did not use annotations and students who used a Web-based annotation system for learning material were compared. By using a questionnaire, they found that most of the students agree that the annotation system improved their online reading performances and was easy to use; furthermore, using the annotation system improved the interaction between learners and the provided materials, by increasing students' interest in learning; finally, students reported that the possibility of sharing annotations both between groups and publicly improved their motivation to learn. In addition, [46] conducted a series of statistical analyses to determine the impact of the use of annotations in different learning scenarios, namely individual reading, group annotation sharing, public annotation sharing, and final examination. They report that, in general, the use of annotations can raise a student's learning achievements in most scenarios, even though in the final examination scenario the high motivation of students to pass the exam produced similar results with and without using annotations. Finally, [46] note a positive correlation between the quantity of 
annotations made and the learning achievements: the greater the number of annotations, the higher the learning achievements. Interestingly enough, they discovered that this effect is more prominent in the group annotation sharing scenario with respect to the public annotation sharing scenario and they argue that this is due to the fact that public annotations may reduce learner's desire to make their own annotations.

\subsection{Historical Viewpoint}

In order to give an idea of the purport of the annotation in our cultural heritage, we discuss how the gloss, a particular kind of annotation, has been used with the passing of time and what the impacts of its use have been. A full discussion on the historical viewpoint on annotations can be found in [5].

The word gloss derives from the ancient Greek word $\gamma \lambda \tilde{\omega} \sigma \sigma \alpha$ (glôssa), that means tongue, language, idiom, spoken word, foreign or obsolete word [44, p. 620] and [62, p. 393].

As reported in [48, pp. 652-653], at the time of the ancient Greeks, the term gloss meant an obscure, archaic, dialect, or rare locution that required an additional explanation. These locutions were the object of study by grammarians or the object of research by scholarly poets, especially the Alexandrian poets, who enriched their compositions with these terms. Therefore, gloss meant the explanations themselves of such locutions, either collected in wide-ranging lexicons or as interlinear notes placed above the words to be explained. This methodology of study and a lexicographical practice dates back to ancient times (there were glosses to Homer as early as the V century B.C.) and was fully developed by the grammarians of the Alexandrian age. During the Bizantine age and the Middle Ages, the term gloss meant an interlinear or marginal note to a biblical or juridical codex. For the biblical codices, the gloss was a very short paraphrase to explain a passage of the Bible, sometimes together with a mention to its allegorical interpretation. On the other hand, for the juridical codices, the glosses were explanatory annotations which constituted a thorough commentary to the text.

The gloss was a practice that flourished especially in the juridical context, as reported by [47, pp. 427-429]. During the Roman Empire, one of the usual literary forms of Roman jurisprudence was the comment to the works of former jurists, so that it is often possible to distinguish the annotated text from the annotation to the text; furthermore, the glosses were sometimes physically separated from the annotated text. However, the most famous use of this kind of method of study is credited to the Bolognese school: indeed, the word gloss denoted the way of studying the Justinian Code practised in Bologna, which began in the 12 th century A.D.. The Bolognese gloss passed from a simpler form to a more complex one by passing from simple interlinear notes to a real theoretical treatment of the subject. The glossarist reveals the contradictions (contrarietates) of the Justinian books, raises doubts $(d u$ biationes or dubietates), which often give rise to controversies (dissensiones). 
The contradictions often find an explanation (solutio) and the doubts disappear by means of an appropriate distinction (distinctio or differentia). The glossarist teaches the Justinian books and creates cases in point and examples that originate glosses pointing out the different cases (casus); furthermore, the glossarist establishes and defines rules derived from the texts he studies, and, accordingly, creates glosses that report such rules (regulae) and definitions (definitiones). In conclusion, the Bolognese gloss was a way of doing research aimed at defining and elucidating the law.

The glosses were usually arranged on a page around the text, as shown in Fig. 1, which reproduces a page from the work Ars notariae (Handbook for notaries) written by Salatiele, one of the professors at the University of Bologna in the Middle Ages [36,63].

The intellectual work entailed by the gloss is of very high quality, because it is a method of both study and research. This kind of intellectual work gives

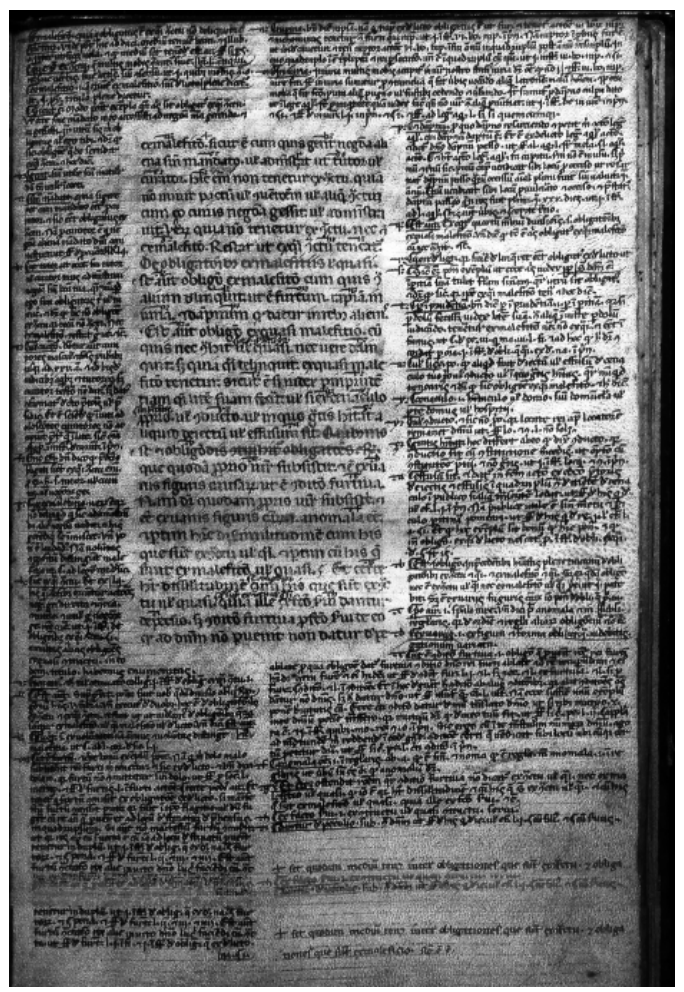

Fig. 1: The photo shows the typical structure of the gloss: the author's commentary, written in smaller characters, is placed around the text, written in greater characters (Italia, Bologna, Biblioteca Comunale dell'Archiginnasio, Salatiele, Ars notariae, ms B 1484, c. 12r - published with the permission of Biblioteca Comunale dell'Archiginnasio di Bologna - taken from [5]) 
us an idea of how strong the active involvement required by the gloss is: it does not concern only the authors themselves, but it is also capable of involving and stimulating a wide community of people who work, study and do research on a subject. Therefore, it turns out that an annotation may comprise a public dimension, because it becomes the vehicle for carrying and transmitting ideas and knowledge to other people, or it may comprise a shared dimension only, if the recipients of the annotation are less numerous.

The research or study aspects and the public or shared dimension entailed by the gloss help us to understand how long-lasting and recordable the annotations are. Indeed, not only are they comments and remarks to a text, but also an autonomous intellectual work which is worth recording.

This important lesson learned from the past should also be taken into account when we design an annotation management system, because the possibility of digitally annotating and quoting can be a valid support to the research work in a networked and distributed environment.

\subsection{System Viewpoint}

This section discusses the two main approaches that have been adopted to deal with annotations when designing an annotation management system: we can consider them as either metadata or content.

Table 1 summarizes and presents systems along two dimensions. One is the degree of structure of the content and the other is the degree of structure of the annotation. The structure of the content can range from loosely structured documents, as in the case of the Web, to highly structured data, as in the case of a database ${ }^{1}$. Similarly, the structure of the annotation can vary from unstructured or loosely structured annotations, as in the case of content annotations, to very structured annotations, as in the case of metadata annotations.

When annotations are considered as metadata, they are additional data which relate to an existing content and clarify the properties and semantics of the annotated content. With this aim in mind, annotations have to conform to some specifications which define the structure, the semantics, the syntax, and even the values that annotations can assume. The recipients of this kind of annotation are both people and computing devices. On the one hand, metadata can benefit people because, if they are expressed in a human-readable form and their format and fields are known, they can be read by people and used to obtain useful and well-structured information about an existing content. On the other hand, metadata offer computing devices the means for automatically processing the annotated contents.

In the Web, the most relevant example of this kind of annotations is the Semantic Web $[43,70]$, which aims at enhancing human-understandable data,

\footnotetext{
${ }^{1}$ Note that we have put the NoteCards system under the Web box, even though it was developed before the Web, because a hypermedia system is much more closer to the Web than to DBMSs or DLMSs.
} 
Table 1: Summary of the different viewpoints about annotations with respect to their use in the Web, DLMSs, and DBMSs

\begin{tabular}{|c|c|c|c|}
\hline & \multicolumn{2}{|c|}{ increasing structure of the annotation } \\
\hline & & Content & Metadata \\
\hline \multirow{3}{*}{ 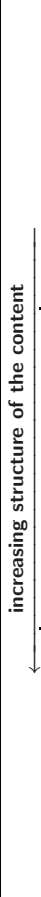 } & Web & $\begin{array}{c}\text { CoNote } \\
{[32]} \\
\text { MADCOW } \\
{[24,25]} \\
\text { NoteCards } \\
{[41,42]}\end{array}$ & $\begin{array}{c}\text { Annotea } \\
{[50,69]} \\
\text { Semantic Web } \\
{[43,70]}\end{array}$ \\
\hline & DLMS & $\begin{array}{c}\text { COLLATE } \\
{[34,67]} \\
\text { DiLAS } \\
{[2,14,19]} \\
\text { FAST } \\
{[8,10-12]}\end{array}$ & $\begin{array}{c}\text { IPSA } \\
{[3,16,17]} \\
\text { SCHOLNET } \\
{[31]}\end{array}$ \\
\hline & DBMS & & $\begin{array}{c}\text { BIODAS } \\
{[66]} \\
\text { Data Provenance } \\
{[23,27,28,40]}\end{array}$ \\
\hline
\end{tabular}

namely Web pages, with computer-understandable data, namely metadata, and this process is called semantic annotation. The Annotea project [50,69] represents a first step in the direction of creating a shared metadata infrastructure.

In the context of Digital Library Management Systems (DLMSs), SCHOLNET [31] uses annotations as metadata to support communication and interaction within scholarly communities by introducing a semantic annotation model, where annotations are treated as documents themselves, the semantics of which is captured by a controlled vocabulary of annotation types. In a similar context, IPSA $[3,16,17]$ supports the annotation and personalization of a digital archive of images taken from illuminated manuscripts [29]. The goal is supporting the research on illuminated manuscripts which unveils hidden connections between illustrations belonging to different manuscripts. In IPSA annotations are links that connect one image to another and they are 
drawn from a link taxonomy which specifies the relationship between the two images.

Annotations are also used in the context of DataBase Management Systems (DBMSs) and, in particular, in the case of curated databases and scientific databases. For example, BIODAS [66] provides a distributed annotation system, which is a system based on Web servers for sharing lists of annotations across a certain segment of the genome. In this context, annotations are often employed for addressing the wider problem of data provenance which is the description of the origins of a piece of data and the process by which it arrived in a database $[27,28]$. Data provenance is undoubtedly an open and challenging research issue in the field of DBMSs, as [1] points out, and annotations have different applications in this regards: tracing the provenance and flow of data, reporting errors or remarks about a piece of data, and describing the quality or the security level of a piece of data [23]. Recently, [40] uses annotations in interactive visualization systems as a means for both capturing the history of user interaction with the visualization system and keeping track of the observations that a user may make while exploring the visualization.

When annotations are regarded as additional content which relates to an existing content, they increase the existing content by providing an additional layer of elucidation and explanation. However, this elucidation does not happen, as in the case of annotations as metadata, by means of some kind of constrained or formal description of the semantics of the annotated object. On the contrary, the explanation itself takes the shape of an additional content which can help people understand the annotated content. However, the semantics of the additional content may be no more explicit for a computing device than the semantics of the annotated content. This view of annotations is comparable to the activity of reading a document and adding notes to it: explanation and clarification of words or passages of the document by expounding on it, providing a commentary on it, and finally completing it with personal observations and ideas.

Therefore, the final recipients of this kind of annotation are people, because a content annotation does not make the annotated object more readily processable by a computer than the same object without annotations. In fact, from the point of view of a computer, the semantics of content annotations needs to be in some way processed, e.g. indexed, before it can be used to deal with the semantics of the annotated object; this is quite different from the case of metadata annotations, which are pieces of information ready to be used for interpreting the semantics of the annotated object. In contrast, the additional semantics provided by content annotations can offer people useful interpretations and comments for the annotated object, making it easier to understand its hidden facets.

In the field of hypermedia/hypertext systems, NoteCards $[41,42]$ is a system designed for helping people to work with ideas: authors, researchers, and intellectual work practitioners can analyze information, construct models, formulate topics, and elaborate ideas by using a network of electronic notecards 
interconnected by typed links. One of the famous "seven issues" mentioned by [41] concerns support for collaborative work: he highlighted how annotations are part of the "activities that form the basis of any collaboration effort" [41, p. 848]. Moving forward in the context of the Web, the CoNote [32] is a cooperative system for supporting communications within groups of users by using shared annotations on a set of documents. CoNote offers plain text or HyperText Markup Language (HTML) [68] annotations on Web pages and pays particular attention in structuring annotations on the same part of a document as a tree, in order to ease the discussion among the users by supporting replies to previously inserted annotations. Finally, a recent example of this kind of annotation system in the Web is Multimedia Annotation of Digital Content Over the Web (MADCOW) [24,25], which enables multimedia annotation on Web pages and is based on a client-server architecture.

As an example of this use of annotations in DLMSs, Collaboratory for Annotation Indexing and Retrieval of Digitized Historical Archive Material (COLLATE) [34,67] supports the collaboration among film scientists and archivists who are annotating historical film documentation, dealing with digitized versions of documents about European films from the 1920s and 1930s. In COLLATE annotations are dialog acts, part of a discourse about film documentation, and these constitute the document context, intended as the context of the collaborative discourse in which the document is placed. Flexible Annotation Service Tool (FAST) [8-12] is a flexible system designed to support its integration into a wide range of different DLMSs. Annotations in FAST allow users to merge their personal content with the information resources managed by diverse DLMSs: annotations can span and cross the boundaries of a single DLMS, annotating digital objects that are part of different digital libraries. In this way, by using annotations, users may link digital objects that otherwise would have remained separated because they are managed by different DLMSs. [49] recently noted this as an advantage for users and a challenge for the next generation DLMSs. Finally, FAST also constitutes the underlying infrastructure of the Digital Library Annotation Service (DiLAS) project $[2,14,19]$, which is an ongoing project in the framework of DELOS, the European Network of Excellence on Digital Libraries. The goal of DiLAS is to design and develop a generic annotation service which can be easily used in different DLMSs; the annotation service is being evaluated as a new way of interacting with a Digital Library $(D L)$ and cooperating among DL users and stakeholders.

Summing up, the final recipients of annotations can be computing devices and people. The former is mainly the case of metadata annotations which allow annotated objects to be automatically processed, integrated and reused in different applications, even though these metadata annotations can be understandable and useful for people too. The latter is mainly the case of content annotations which elucidate and expound on an annotated object. Note that, also in this latter case, a computing device can become the recipient of such annotations, provided that some further step of processing is performed, 
e.g. indexing. However, in both cases, the semantics of the annotation itself needs to be taken into consideration and modeled. This can happen formally and precisely by agreeing on metadata standards which describe how annotations should be interpreted and used; alternatively, support can be provided for identifying different pre-defined annotation types, perhaps with varying levels of detail.

The medium of the annotation can vary a lot: it can range from textual annotations, to image, audio, and video annotations; in a general setting, we may need to deal with multimedia rich annotations, composed of different parts, each with its own medium. All of these different kinds of media have to be considered and properly modeled, in a uniform way where possible.

Both annotations and annotated objects need to be uniquely identified. Moreover, annotations comprise a temporal dimension that is often not explicit, but which limits the creation of the annotation to the existence of another object. This temporal relationship between the annotation and the annotated object does not mean that the annotation cannot be considered a stand-alone intellectual work, but it does impose a temporal ordering between the existence of an annotated object and the annotation annotating it which cannot be overlooked. In addition, once we have identified both the annotation and the annotated object, we need to link and anchor the annotation to the part of the annotated object in question. This can happen in a way that mainly depends on the medium of the annotated object. On the whole, we need to model how annotations and annotated objects are uniquely identified and linked together, maybe with a varying degree of granularity in the anchoring, paying particular attention to the temporal dimension that regulates the relationships between annotations and annotated objects.

As far as co-operation is concerned, almost all of the analyzed systems show that annotations have great potential for supporting and improving interaction among users, and even among computing devices. Therefore, there is a need for modeling and offering different scopes of annotations, e.g. private, shared, or public, and managing the access rights of various groups of users.

Finally, a relevant aspect of annotations is that they can take a part of a hypertext $[15,41,52]$ since they enable the creation of new relationships among existing objects, by means of links that connect annotations together with existing objects, as we will see later in more detail. The hypertext viewpoint about annotations is common to different systems, such as Annotea, MADCOW, and NoteCards in the hypermedia/Web context, or DiLAS, FAST, and IPSA in the DLMS context. [41] points out that annotations are one of the activities that form the basis of any collaborative effort and for which hypermedia systems are ideally suited, while [52] considers annotations a natural way of creating and growing hypertexts that connect information resources by actively engaging users. 


\section{Overview of the Formal Model}

In this section we provide a description and overview of the formal model, explaining the main concepts around which it is build, and give an idea of its possible application areas and the next steps we can undertake by using it as a starting point.

\subsection{Modeling Approach}

Figure 2 provides both an overview of the areas covered and the detail of the definitions introduced within each area. The figure clearly shows how these areas correspond to the very basic issues that emerge when we think about annotations: we need to identify annotations and annotated objects in order to link them together, perhaps providing facilities for supporting cooperation, and we have to deal with both the actual contents of an annotation and the semantics expressed by those contents.

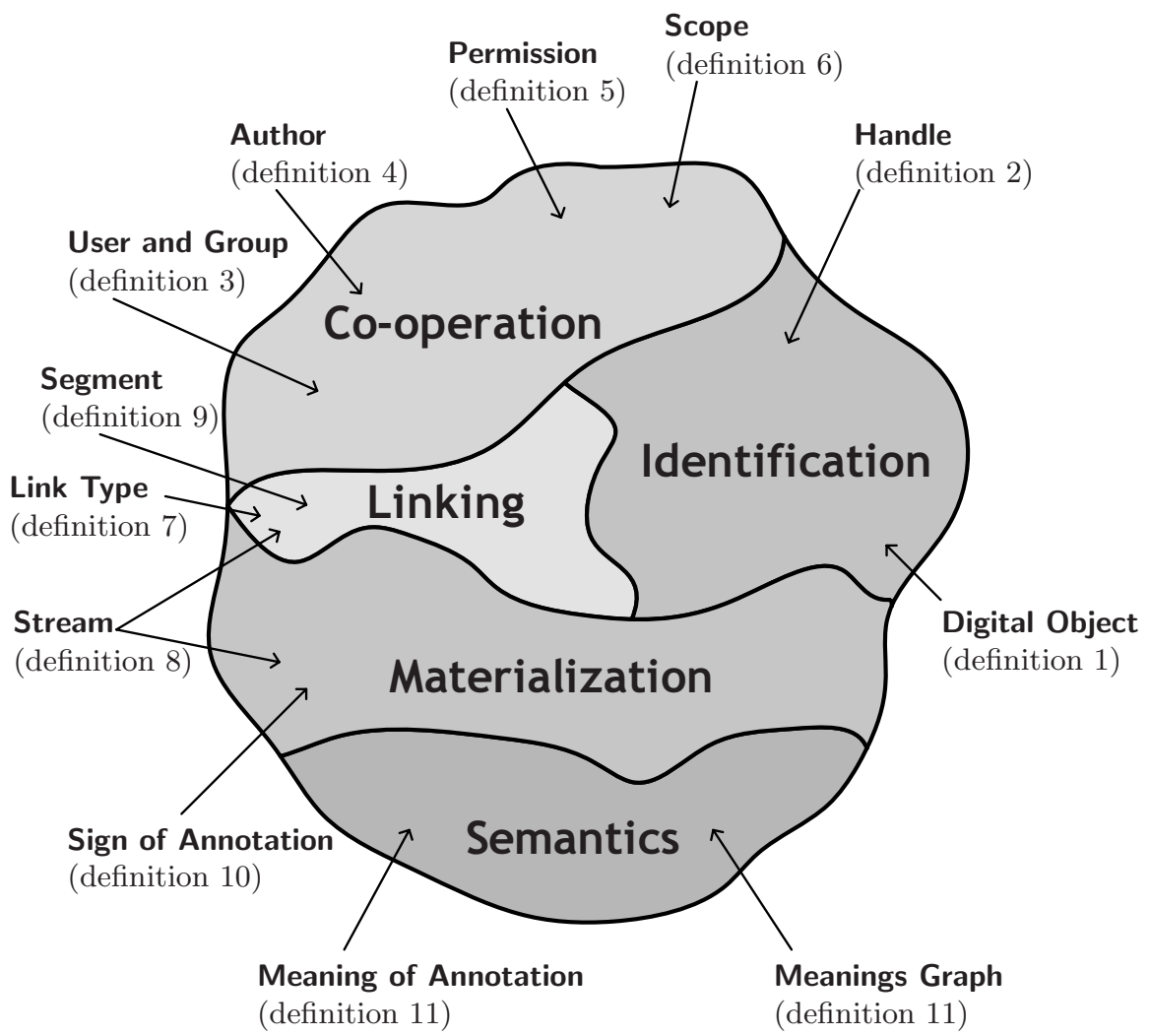

Fig. 2: Overview of the areas covered by the proposed formal model of the annotation - from [13] 
In the remainder of this section, we will briefly introduce the areas shown in Fig. 2, which can be used as a map of the concepts dealt with in the formal model.

Identification This is the problem of uniquely identifying both the annotation and the annotated objects, highlighting the temporal constraints between them. This area is built around the concept of handle, which is defined as a unique identifier of both digital objects and annotations, and the proposed notation for dealing with the time dimension involved by annotations.

Co-operation This is about annotations as a co-operation tool among users. It introduces the definitions of user and group of users, together with the associated concept of scope of annotation and access permission, which regulate the access policies for a given annotation.

Linking This deals with the allowed linking patterns between digital objects and annotations, and the problem of correctly anchoring annotations to digital objects. It defines the concepts of link type, which is defined as the allowed methods of linking annotations to annotated objects, stream, which abstracts the notion of content of a digital object, and segment, which represents a given portion of a stream, useful for anchoring an annotation to a digital object.

Semantics This concerns the meaning of the annotation and what it stands for, trying to make explicit the semantics of the different parts of the content of an annotation. It introduces the notions of meaning of annotation, which is part of the semantics of the whole annotation, and meanings graph, which is a graph which allows for interoperability between the different meanings.

Materialization This deals with the way in which the semantics carried by an annotation can take shape, that is, the actual content of the annotation perceived by the user. It describes the sign of annotation, which is a particular type of stream representing part of the content of an annotation.

Finally, we will discuss the hypertext between annotated objects and annotations, which is build on top of the previously introduced concepts and can also be thought of as a kind of concise view of the main relationships between annotations and annotated objects.

\subsection{Applications and Uses of the Formal Model}

A first application of the formal model is to use it as an analysis tool for studying and having a better comprehension of an existing annotation management system. Indeed, the main areas identified above act as main questions which can be asked about the annotation management system: which mechanism is used for identifying annotations and annotated objects? Which kind of support is offered for the co-operation of the users and which degree of visibility for annotations are available? How are annotations linked to annotated objects? Are they embedded in the annotated objects or kept separated? What is 
the granularity available for anchoring an annotation to an annotated object? How is the semantics of an annotation expressed, by using fixed annotation types or a more flexible mechanism? What about the content of the annotation: can different media be mixed together? All these question and even more detailed ones can be derived from the formal model and can be useful for analysing and comparing annotation management systems in a qualitative way.

A step further is to apply the definitions of the model to describe an annotation management system. In this way, we can formally compare different annotation management systems and spot differences and commonalities, by using the model as a kind of landmark which allows us to determine the features of each system.

Another interesting possibility is to use the formal model as the starting point for defining and creating more refined and specialized models, which may be more tailored to specific applicative contexts. In this context, the formal model provides a kind of template which outlines the main areas and concepts which can be further specialized. In addition, the formal model ensures the comparability and compatibility of the derived models. In this way, we avoid the flourishing of unrelated annotation models, as has happened to date, while maintaining both the coherence among different specialistic annotation models and the possibility of passing from one to another in a clearly defined way.

We successfully applied the above mentioned strategy in the context of illuminated manuscripts [17,18]. Image digital archives of illuminated manuscripts can become a useful tool for researchers in different disciplines and we proposed to provide them with tools for annotating images to disclose hidden relationships between illustrations belonging to different works. Relationships can be modeled as typed annotative links, which induce a hypertext over the archive. In this context, we defined a model for annotations which is derived from the one presented here and represents the basis for building methods for automatically processing existing relationships among link types and exploiting the properties of the graph which models the hypertext between annotations and annotated images.

The hypertext between annotations and annotated objects can be exploited for providing alternative navigation and browsing capabilities. In particular, DLMSs usually offer some basic hypertext and browsing capabilities based on the available structured data, such as authors or references. On the other hand, DLMSs do not normally provide users with advanced hypertext functionalities, where the information resources are linked on the basis of the semantics of their content. Therefore, annotations can turn out to be an effective way of associating this kind of hypertext to a DLMS to enable the active and dynamic use of information resources. In addition, this hypertext can span and cross the boundaries of the single DLMS, if users need to interact with the information resources managed by diverse DLMSs $[9,10]$. This latter possibility is quite innovative, because it offers the means for interconnecting 
various DLMSs in a personalized and meaningful way for the end-user, and, as [49] point out, this is a major challenge for the next generation DLMSs.

Moreover, the proposed formal model constitutes the necessary groundwork for designing and formalizing search algorithms and expressing query languages which take annotations into account in order to retrieve more and better ranked objects in response to a user query. Indeed, annotations provide us with an additional source of evidence, which is complementary to that already contained in the set of documents. Therefore, we can exploit annotations with the two final goals of retrieving more relevant documents and ranking them better. Furthermore, the paths that connect annotations to documents become the vehicle for moving this further source of evidence towards the documents. In this context, the hypertext between annotations and annotated objects is the basic infrastructure for combining the sources of evidence which come from annotated objects and annotations. We have already started to work on this problem in the context of data fusion $[11,12]$. This is because we need to combine the source of evidence which comes from annotations with the source which comes from documents. For the future, we plan to employ both hypertext information retrieval $[6,20]$ and link fusion techniques [71] for designing advanced search algorithms which involve annotations based on our formal model.

Once we have developed search strategies that exploit annotations, we will therefore need to evaluate their retrieval performances by using standard information retrieval methodologies. We plan to adopt the Cranfield methodology [30], which makes use of experimental collections to measure the performances of an information retrieval system. The performances are measured by using the standard precision and recall figures [61,64], but according to [10] we also need a statistical methodology for judging whether the measured performances can be considered statistically significant. The next step will be to investigate the possibility of using measures that differ from precision and recall and are better tailored to the features of annotations. Finally, there is a lack of experimental test collections with annotated digital contents. We have already started to work on this problem [7] and the future research work will also concern the design and development of this kind of test collection.

Finally, the formal model provides us with sound bases for designing and developing an annotation service which can be easily integrated into a wider IMS. Indeed, a clear definition of the concepts related to the annotation allows us to modularly design the functionalities of such service. In particular, we can separate the functionalities needed to manage, access, and search annotations, which constitute the core of an annotation service, from the functionalities needed to integrate such annotation service into an IMS. In addition, the formal model can be used as a starting point for deriving conceptual and logical models of the annotation suitable for managing the persistence of the annotations, for example, by using a DBMS.

We successfully adopted this approach for designing and developing the FAST annotation service, whose conceptual and logical model of 
annotation $[15,16]$ has been derived from our formal model, which is independent from any particular IMS [10], offers advanced search functionalities based on annotations $[11,12]$, and has been successfully integrated into different systems $[2,4]$.

\section{The Formal Model}

\subsection{Identification}

In order to uniquely identify both the annotation and the annotated objects, we need to proceed as follows: firstly, we need to define the objects we deal with; then, we also have to be able to deal with objects whose relationships are constrained by a temporal dimension; finally, a suitable identification mechanism has to be provided.

\section{Document, Annotation, and Digital Object Sets}

According to widely accepted terminology, we adopt the term digital object to refer to information resources managed by an IMS. In the following, we need terminology to distinguish between two kinds of digital objects: the generic ones managed by the IMS, which we call documents, and the ones that are annotations. Therefore, when we use the generic term digital object, we mean a digital object that can be either a document or an annotation. The following definition introduces the different sets of digital objects we will need to deal with.

Definition 1. Let us define the following sets:

- $D$ is a set of documents and $d \in D$ is a generic document. $U_{D}$ is a universe set of documents, which is the set of all the possible documents, so that $D \subseteq U_{D}$.

- $A$ is a set of annotations and $a \in A$ is a generic annotation. $U_{A}$ is a universe set of annotations, which is the set of all the possible annotations, so that $A \subseteq U_{A}$.

- $D O=D \cup A$ is a set of digital objects and do $\in D O$ is either a document or an annotation. $U_{D O}=U_{A} \cup U_{D}$ is a universe set of digital objects, so that $D O \subseteq U_{D O}$.

\section{Expressing the Temporal Dimension Involved by Annotations}

The universe sets $U_{D}, U_{A}$, and $U_{D O}$ are abstract sets, since they contain all the possible needed objects, whether they actually exists or not in any given moment; on the other hand, the sets $D, A$, and $D O$ are tangible sets that contain the objects that already exist in a given moment: if we pick out an 
element from $D, A$, or $D O$ we are dealing with a digital object that has been created even before we start working on it; in other words, the element already exists. The $D, A$, and $D O$ sets can be considered time-variant sets, since we can add, delete or modify elements of these sets over time. On the other hand, the $U_{D}, U_{A}$, and $U_{D O}$ sets can be considered time-invariant sets, since they already contain every possibile object we may need to deal with.

The annotation is the result of an intellectual task performed on an existing digital object and it follows an already existing digital object. Therefore, the annotation comprises a temporal dimension which is often not explicit, but which limits the creation of the annotation to the existence of another digital object. This temporal relationship between the annotation and the annotated digital object does not mean that the annotation cannot be considered a stand-alone intellectual task, but it does impose a temporal ordering between the existence of an annotated digital object and its annotation which cannot be overlooked.

In conclusion, we need some mechanism for rendering the time dimension explicit, if necessary. We will illustrate this mechanism by means of an example concerning the creation of a new digital object. Although, this example makes use of the set $D O$, it does have a more general validity.

The creation of a new digital object consists of the following events:

1. we start with the set of digital objects at time $k: D O(k)$;

2. we create a new digital object, that is, we pick out an element from the universe set of digital objects that does not belong to $D O(k):$ do $\in \overline{D O}(k) \subseteq$ $U_{D O}$

3. we end up with a new set of digital objects at time $k+1$, which contains the newly created digital object: $D O(k+1)=(D O(k) \cup\{d o\}) \in 2^{U_{D O}}$.

Therefore, we have the following temporal ordering:

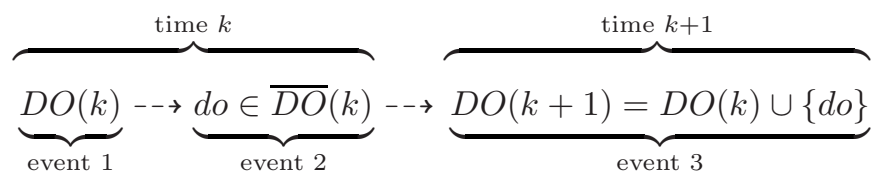

both events 1 and 2 happen at time $k$, but at that time the newly created digital object does not yet belong to the set $D O(k)$ of digital objects at time $k$; event 3 happens at time $k+1$ and represents the new set of digital objects that now also contains the newly created and existing digital object.

$D O(k)$ and $D O(k+1)$ unambiguously identify the digital objects we are dealing with: the newly created digital objects are given by $D O(k+1) \backslash D O(k)$. Therefore, we can talk about the digital objects identified by the transition from $D O(k)$ to $D O(k+1)$. We assume that the operations previously shown are atomic, i.e. no operation can occur during the execution of another operation, so as to avoid concurrency issues.

In conclusion, this mechanism provides us with a means to clearly identify which objects are involved in a given operation, when they can be utilized, 
and the ordering among the different events involved by an operation. For a more detailed discussion on this approach, please refer to [13].

In the following sections, we will use the notation $D O(k)$, which explicitly points out the time dimension, only when needed; otherwise we will use the simpler notation $D O$, without explicitly pointing out the time dimension. We will also use a similar notation for the other sets we will define below.

\section{Handle}

According to the previous discussion, we can assume that each digital object is identified by a unique handle, which is a name assigned to a digital object to identify and to facilitate the referencing process to the digital object. Over the past years, various syntaxes, mechanisms, and systems have been developed to provide handles or identifiers for digital objects: Uniform Resource Identifier (URI) [21,22], Digital Object Identifier (DOI) [59]; OpenURL [57]; Persistent URL $(P U R L)^{2}$; and PURL-based Object Identifier $(P O I)^{3}$.

The following definition introduces the notion of handle, compatible with the mechanisms described above, and its relationship with digital objects.

Definition 2. $H$ is a set of handles such that $|H|=|D O|$ and $h \in H$ is a generic handle. $U_{H}$ is a universe set of handles, which is the set of all the possible handles, such that $\left|U_{H}\right|=\left|U_{D O}\right|$; it follows that $H \subseteq U_{H}$.

We define a bijective function $\mathrm{h}: U_{H} \rightarrow U_{D O}$ which maps a handle to the digital object identified by it:

$$
\forall d o \in U_{D O}, \exists ! h \in U_{H} \mid \mathrm{h}(h)=d o \Rightarrow \mathrm{h}^{-1}(d o)=h
$$

The relationship between the sets $H$ and $U_{H}$ is the same as the one between the sets $D O$ and $U_{D O}$, described in Sect. 4.1 .

\subsection{Co-operation}

In order to provide users with annotations as an effective co-operation tool, we need to proceed as follows: firstly, we need to define the notion of user, group of users, and author; then, we have to deal both with different scopes of annotation, and various access permissions.

\section{User, Group of Users and Author}

Definition 3. Let $U S R$ be a set of users and usr $\in U S R$ is a generic user; $U_{U S R}$ is a universe set of users, which is the set of all the possible users, so that $U S R \subseteq U_{U S R}$.

\footnotetext{
${ }^{2}$ http://purl.oclc.org/

${ }^{3}$ http://www.ukoln.ac.uk/distributed-systems/poi/
} 
$G R \subseteq 2^{U S R}$ is a set of groups of users and $G \in G R$ is a generic group of users; $U_{G R}=2^{U_{U S R}}$ is a universe set of groups of users, which is the set of all the possible groups of users, so that $G R \subseteq U_{G R}$.

We define a function gr $: U S R \rightarrow 2^{G R}$ which maps a user to the groups of users he belongs to. The following constraint must be adhered to:

$$
\forall u s r \in U S R, \operatorname{gr}(u s r) \neq \varnothing
$$

i.e. each user in USR must belong to at least one group of users.

The relationship between the sets $U S R$ and $G R$ and the sets $U_{U S R}$ and $U_{G R}$ is the same as the one between the sets $D O$ and $U_{D O}$, described in Sect. 4.1.

Digital objects - both documents and annotations - always have at least one author who authored them. Therefore, the author is a specialization of the more general concept of user, introduced in the definition above, i.e. an author is a user who authored one or more digital objects.

Definition 4. Let us define a function au : USR $\rightarrow 2^{H}$ which maps a user to the handles of the digital objects authored by him. Let the set of authors $A U$ be the following set:

$$
A U=\{u s r \in U S R \mid \operatorname{au}(u s r) \neq \varnothing\}
$$

we denote with au $\in A U \subseteq U S R$ a generic author. The following constraint must be adhered to:

$$
\forall h \in H \exists a u \in A U \mid h \in \mathrm{au}(a u)
$$

i.e. each digital object must be authored by at least one author.

The function au characterizes the authors, distinguishing them from generic users; indeed, if a generic user usr $\in U S R$ has not authored any digital object, it follows that $\mathrm{au}(u s r)=\varnothing$ and thus usr $\notin A U$.

\section{Permission}

An annotation can have different access permissions, as introduced in the following definition.

Definition 5. Let $P=\{$ Denied, ReadOnly, ReadWrite $\}$ be a set of access permissions and $p \in P$ is an access permission. Let us define the following relations:

- equality relation $=$

$$
\begin{aligned}
\{(p, p) \in P \times P \mid p \in P\}=\{ & (\text { Denied, Denied }),(\text { ReadOnly, ReadOnly }), \\
& (\text { ReadWrite, ReadWrite })\}
\end{aligned}
$$


- strict ordering relation $\prec$

$\{($ Denied, ReadOnly $),($ Denied, ReadWrite $),($ ReadOnly, ReadWrite $)\}$

- ordering relation $\preceq$

$$
\left\{\left(p_{1}, p_{2}\right) \in P \times P \mid p_{1}=p_{2} \vee p_{1} \prec p_{2}\right\}
$$

In contrast to the set of the previous definitions, the set of access permissions $P$ is a time-invariant set which does not need the notation for taking into account the temporal dimension. Indeed, we assume that an annotation can only have the access permissions listed above. Note that $(P, \preceq)$ is a totally ordered set.

\section{Scope}

An annotation can have one of the following scopes, as introduced in the following definition.

Definition 6. Let $S P=\{$ Private, Shared, Public $\}$ be a set of scopes and $s p \in S P$ is a scope. Let us define the following relations:

- equality relation $=$

$$
\begin{aligned}
\{(s p, s p) \in S P \times S P \mid s p \in S P\}=\{ & (\text { Private, } \text { Private }),(\text { Shared, Shared }), \\
& (\text { Public }, \text { Public })\}
\end{aligned}
$$

- strict ordering relation $\prec$

$$
\{(\text { Private, Shared }),(\text { Private, Public }),(\text { Shared, Public })\}
$$

- ordering relation $\preceq$

$$
\left\{\left(s p_{1}, s p_{2}\right) \in S P \times S P \mid s p_{1}=s p_{2} \vee s p_{1} \prec s p_{2}\right\}
$$

As in the case of the set of access permissions, the set of scopes $S P$ is also a time-invariant set, because we assume that an annotation can have only one of the three scopes listed above. Note that $(S P, \preceq)$ is a totally ordered set.

\subsection{Linking}

In order to link annotations to digital objects and to correctly anchor annotations to digital objects, we need to proceed as follows: firstly, we need to choose a linking mechanism and define the link types that can exist between annotations and digital objects; then, since annotations are usually linked to specific parts of a digital object, we need to model the content of digital objects; finally, a suitable anchoring mechanism for annotations has to be provided. 


\section{Linking Annotations to Digital Objects}

Handles can be used not only for the purpose of uniquely identifying a digital object, but they can also provide us with a means for linking an annotation to a digital object. This use of handles is particularly clear if we think about Uniform Resource Locators (URLs), but it is also still valid in the case of the other types of handles presented in Sect. 4.1.

Once we have decided to use handles as basic mechanism for linking annotations to digital objects, we still have to consider the kind of links an annotation can have with a digital object. Annotations can be linked to digital objects with two main types of links:

- annotate link: an annotation annotates a digital object, which can be a document or another annotation.

The "annotate link" is intended to allow an annotation only to annotate one or more parts of a given digital object. Therefore, this kind of link lets the annotation express intra-digital object relationships, meaning that the annotation creates a relationship between the different parts of the annotated digital object;

- relate-to link: an annotation relates to a digital object, which can be a document or another annotation.

The "relate-to link" is intended to allow an annotation only to relate to one or more parts of other digital objects, but not the annotated one. Therefore, this kind of link lets the annotation express inter-digital object relationships, meaning that the annotation creates a relationship between the annotated digital object and the other digital objects related to it.

With respect to these two main types of link, we introduce the following constraint:

an annotation must annotate one and only one digital object, which can be either a document or another annotation, i.e. an annotation must have one and only one "annotate link".

This constraint means that an annotation can be created only for the purpose of annotating a digital object and not exclusively for relating to a digital object. An annotation, then, can annotate one and only one digital object, because the "annotate link" expresses intra-digital object relationships and thus it cannot be mutual to multiple digital objects different from the annotated one. Finally, this constraint does not prevent the annotation from relating to more than one digital object, i.e. from having more than one "relate-to link".

Definition 7. Let LT be a set of link types; an element $l t \in L T$ corresponds to one of the allowed link types. The set LT contains the following link types: $L T=\{$ Annotate, RelateTo $\}$.

As in the case of the set of access permissions and the set of scopes, the set of link types $L T$ is a time-invariant set too, because we assume that an 
annotation can be linked to digital objects only with the link types listed above.

\section{Stream}

Digital objects can be very different - texts, images, audio, video, hypertexts, multimedia objects, and so on - and the way in which their structure and content is modeled and expressed can also vary widely across different conceptual and logical models of IMS and digital object. Nevertheless, many of these types of models share the idea that beyond representing the structure of the digital object the model also has to take into account a mechanism for representing the actual content of the digital object.

The following definition introduces the concept of stream in order to represent the actual content of a digital object or a part of it. The definition of stream is inspired by $[39,56]$ but with some differences which will be discussed below.

Definition 8. A stream sm is a finite sequence:

$$
\operatorname{sm}: I=\{1,2, \ldots, n\} \rightarrow \Sigma, \quad n \in \mathbb{N}
$$

where $\Sigma$ is the alphabet of symbols. We allow the existence of an empty stream $\mathrm{esm}=\varnothing . S M$ is a set of streams and $\mathrm{sm} \in S M$ is a stream. $U_{S M}$ is a universe set of streams, that is, the set of all the possible streams. It follows that $S M \subseteq U_{S M}$.

We define a function hsm $: H \rightarrow 2^{S M}$ which maps a handle of a digital object to the streams contained in that digital object. The following constraint must be adhered to:

$$
\forall h \in H, \operatorname{hsm}(h) \neq \varnothing
$$

i.e. each digital object must contain at least one stream, which could also be the empty stream.

The relationship between the sets $S M$ and $U_{S M}$ is the same as the one between the sets $D O$ and $U_{D O}$, described in Sect. 4.1.

\section{Segment}

The handles discussed in Sect. 4.1 may be capable not only of uniquely identifying a digital object, but also of indicating a part of the identified digital object. For example, a URL can point to any given anchor within a HTML document, or we can use an XPath expression to point to a specific element within an eXtensible Markup Language (XML) document. On the other hand, parts of a digital object cannot always be identified with an arbitrary degree of detail; for example, a URL cannot point to a given word of a HTML document, if this word is not marked with an anchor. Therefore, we need some 
further mechanism for identifying parts of a digital object with the necessary degree of detail.

The following definition introduces the notion of segment, which is a mechanism for selecting parts of a stream; this mechanism can be partnered with the handle of a digital object to provide access to a digital object with the necessary degree of detail.

Definition 9. Given a stream $\operatorname{sm}: I=\{1,2, \ldots, n\} \rightarrow \Sigma, n \in \mathbb{N}, \operatorname{sm} \in S M$, a segment is a pair:

$$
s t_{\mathrm{sm}}=(a, b) \mid 1 \leq a \leq b \leq n, \quad a, b \in \mathbb{N}
$$

A stream segment is a restriction $\mathrm{sm}_{\mid[a, b]}$ of the stream $\mathrm{sm}$ to interval $[a, b]$ associated with the segment $s t_{\mathrm{sm}} . S T$ is a set of segments and $s_{\mathrm{sm}} \in S T$ is a generic segment; $U_{S T}$ is a universe set of segments, which is the set of all the possible segments, so that $S T \subseteq U_{S T}$.

The relationship between the sets $S T$ and $U_{S T}$ is the same as the relationship between the sets $D O$ and $U_{D O}$, described in Sect. 4.1. Definition 9 resembles the definition of segment provided in $[39,56]$.

All of the introduced concepts, namely handle, stream, and segment, provide us with the formal means needed to deal with the linking and anchoring problem related to annotations. By using a handle $h$ we can link an annotation to a digital object; then, the function $\operatorname{hsm}(h)$ allows us to select the desired stream sm of the digital object identified by $h$, be it a physical or a logical view of the actual content of the digital object; finally, a segment $s_{\mathrm{sm}}$ enables the fine-tuned anchoring of the annotation to the digital object.

\subsection{Materialization}

We call sign of annotation the basic way in which an annotation can take shape, i.e. the way of representing and materializing the semantics of annotation.

Definition 10. A sign of annotation is a stream. $S N \subseteq S M$ is a set of signs of annotation and $\mathrm{sn} \in S N$ is a sign. $U_{S N} \subseteq U_{S M}$ is a universe set of signs of annotation, which is the set of all the possible signs of annotation, so that $S N \subseteq U_{S N}$.

The relationship between the sets $S N$ and $U_{S N}$ is the same as the relationship between the sets $D O$ and $U_{D O}$, described in Sect. 4.1.

Henceforth we will use the term sign of annotation, or briefly stated as sign, to indicate a stream that belongs to an annotation. On the other hand, we will use the term stream to indicate a stream that belongs to a digital object without the need of specifying if the digital object is a document or an annotation. 


\subsection{Semantics}

We call meaning of annotation a main feature of the concept of annotation which identifies conceptual differences within the semantics of the annotation or part of it.

Definition 11. $M$ is a set of meanings of annotations, and $m \in M$ is $a$ generic meaning of annotation.

The meanings graph is a labeled directed graph $\left(G_{M}, 1_{M}\right)$, where $G_{M}=$ $\left(M, E_{M} \subseteq M \times M\right)$ and $1_{M}: E_{M} \rightarrow L_{M}$ with $L_{M}$ set of labels.

The meanings function $\mathrm{m}: S N \rightarrow 2^{M}$ associates each sign of annotation with its corresponding meanings of annotation. The following constraint must be satisfied:

$$
\forall \mathrm{sn} \in S N, \mathrm{~m}(\mathrm{sn}) \neq \varnothing
$$

i.e. each sign of annotation has at least one meaning of annotation.

As in the case of the set of access permissions, the set of scopes and the set of link types $L T$, the set of meanings $M$ is a time-invariant set, because we assume that meanings represent a pre-existing knowledge which does not change over time. Therefore, all the needed meanings of annotation are already elements of the set $M$.

The goal of the meanings graph is to provide structure and hierarchy among the meanings of annotation in order to navigate and browse through them. The relation $E_{M}$ can be constrained in many ways to obtain the necessary structure of meanings, which can represent some domain specific knowledge. The labelling function $l_{M}$ can be further exploited to distinguish different kinds of arcs in the set $E_{M}$ in order to better explain the kind of relationship between two different meanings.

The meanings function allows us to associate each sign of annotation with its corresponding meanings in order to clarify the semantics of the sign. Note that the meanings function is neither injective nor surjective. In conclusion, an annotation is expressed by one or more signs of annotation, which in turn are characterised by one or more meanings of annotation, thus defining the overall semantics of the annotation.

\subsection{Annotation}

We are now ready to introduce the definition of annotation. Summing up the concepts introduced in the previous sections, we can briefly say that an annotation is expressed by one or more signs of annotation, such as a piece of text or some graphic mark, which are the way an annotation takes shape. The semantics of each sign is, in turn, defined by one or more meanings of annotation. With respect to the linking issue, an annotation must annotate one and only one digital object, identified by its handle, while it may relate to one or more digital objects. Lastly, the mechanism introduced in Sect. 4.1 
on how to address the time dimension is now fundamental to properly define the relationship between the annotation and the annotated digital object.

Definition 12. An annotation $a \in A(k)$ is a tuple:

$$
\begin{gathered}
a=\left(h_{a} \in H(k), a u_{a} \in U S R(k-1), G_{a} \in 2^{G R(k-1)} \times P, s p_{a} \in S P,\right. \\
\left.\mathcal{A}_{a} \subseteq S N(k) \times L T \times S T(k) \times S M(k-1) \times H(k-1)\right)
\end{gathered}
$$

where:

- $h_{a}$ is the unique handle of the annotation a, i.e. $\mathrm{h}\left(h_{a}\right)=a$;

- $a u_{a}$ is the author of the annotation a, i.e. $h_{a} \in \mathrm{au}\left(a u_{a}\right)$;

- $G_{a}$ are the groups of users with their respective access permissions for the annotation $a$, specified by the pairs $(G, p)$ with $G \in G_{a}$ and $p \in P$;

- $s p_{a}$ is the scope of the annotation a;

- each n-ple of the $\mathcal{A}_{a}$ relation means that the annotation a by means of a sign in $S N(k)$ and a link type in LT is annotating or relating to a segment in $S T(k)$ of a stream in $S M(k-1)$ of a digital object identified by its handle in $H(k-1)$.

Note that since $\forall \mathrm{sm} \in S M(k-1) \mid \exists \alpha \in \mathcal{A}_{a}, \alpha=\left(\mathrm{sn}, t, \mathrm{st}_{\mathrm{sm}}, \mathrm{sm}, h\right)$ must be $\operatorname{sm} \in \operatorname{hsm}(h)$; in other words, the stream sm must be contained in the digital object identified by the handle $h$.

We introduce the following auxiliary sets to simplify the following discussion:

- the set of the signs of annotation that belong to the annotation a: $S N_{a}=\left\{\mathrm{sn} \in S N(k) \mid \exists \alpha \in \mathcal{A}_{a}, \alpha=\left(\mathrm{sn}, l t, s t_{\mathrm{sm}}, \mathrm{sm}, h\right)\right\}=\operatorname{hsm}\left(h_{a}\right)$

- the set of the handles of digital objects that are subject to the tasks of the annotation a:

$H_{a}=\left\{h \in H(k-1) \mid \exists \alpha \in \mathcal{A}_{a}, \alpha=\left(\mathrm{sn}, l t, s t_{\mathrm{sm}}, \mathrm{sm}, h\right)\right\}$

The following constraints must be adhered to:

1. the annotation a must annotate one and only one digital object, and it cannot also relate to this digital object, hence:

$$
\begin{aligned}
\exists ! h \in H_{a} \mid & \\
& \left(\forall \mathrm{sn} \in S N_{a}, \exists ! \alpha \in \mathcal{A}_{a}, \alpha=\left(\mathrm{sn}, \text { Annotate, } s t_{\mathrm{sm}}, \mathrm{sm}, h\right)\right) \wedge \\
& \left(\nexists \alpha_{1} \in \mathcal{A}_{a}, \alpha_{1}=\left(\mathrm{sn}_{1}, \text { RelateTo, } \text { st }_{\mathrm{sm}_{1}}, \mathrm{sm}_{1}, h\right)\right)
\end{aligned}
$$

2. a sign in $S N_{a}$ cannot relate to more than one digital object, hence:

$$
\begin{aligned}
& \forall \mathrm{sn} \in S N_{A} \mid \exists \alpha_{1}, \alpha_{2} \in \mathcal{A}_{a}, \\
& \quad \alpha_{1}=\left(\mathrm{sn}, \text { RelateTo, st } \mathrm{sm}_{1}, \mathrm{sm}_{1}, h_{1}\right), \alpha_{2}=\left(\mathrm{sn}, \text { RelateTo, st } \mathrm{sm}_{2}, \mathrm{sm}_{2}, h_{2}\right) \\
& \quad \Rightarrow \alpha_{1}=\alpha_{2}
\end{aligned}
$$


3. there is no other annotation $a_{1} \in A(k-1)$ that shares signs of annotation with a, hence:

$$
\nexists a_{1} \in A(k-1) \mid S N_{a} \cap S N_{a_{1}} \neq \varnothing
$$

4. if the annotation $a \in A(k)$ annotates or relates to another annotation $a_{1} \in$ $A(k-1)$, then scope and access permission conflicts have to be avoided. Let us define the conflict detector function, cd : $A(k) \times A(k-1) \rightarrow\{0,1\}$, so that:

$$
\operatorname{cd}\left(a, a_{1}\right)=\left\{\begin{array}{l}
0 \quad \begin{array}{l}
\text { if there are neither scope conflicts nor } \\
\text { access permission conflicts }
\end{array} \\
1 \quad \text { if there are either scope conflicts or } \\
\text { access permission conflicts }
\end{array}\right.
$$

Therefore, the following condition must be satisfied:

$$
\forall h \in H_{a} \mid \mathrm{h}(h)=a_{1} \in A(k-1) \Rightarrow \operatorname{cd}\left(a, a_{1}\right)=0
$$

In conclusion, the first part of the annotation tuple is devoted to providing information about the annotation itself, because it specifies the handle of the annotation, its author, its groups of users with their respective access permissions, its scope, the signs of the annotation, and the link types. On the other hand, the second part of the annotation tuple provides information about the annotated or related digital objects, specifying which segment of which stream of which digital object is being annotated or related to, as shown below (we do not use the time dimension notation for space reasons, as it is not needed for this observation):

$$
a=(\underbrace{h_{a}, a u_{a}, G_{a} \times P, s p_{a}, \mathcal{A}_{a} \subseteq S N \times L T}_{\text {information about the annotation }} \times \underbrace{S T \times S M \times H}_{\text {information about the digital object }})
$$

\subsection{Document-Annotation Hypertext}

As explained in Sect. 3, we consider that existing digital objects and annotations constitute a hypertext. The definition and the properties of this hypertext directly follow from the definition of annotation we provided in the previous sections. Therefore, we can consider the document-annotation hypertext as a kind of view on the set of documents and annotations. The aim is to mask all of the details involved by the definition of the annotation itself, and to provide us with a more abstract representation of the objects we dealt with and of their structural relationships.

Definition 13. The document-annotation hypertext is a labeled directed graph:

$$
\left(H_{d a}=\left(D O, E_{d a} \subseteq A \times D O\right), 1_{d a}\right)
$$


where:

- $D O=A \cup D$ is a set of vertices;

- $E_{d a}=\left\{(a, d o) \in A \times D O \mid \exists \alpha \in \mathcal{A}_{a}, \alpha=\left(\mathrm{sn}, t, s t_{\mathrm{sm}}, \mathrm{sm}, \mathrm{h}^{-1}(d o)\right\}\right.$ is a set of edges;

- $1_{d a}: E_{d a} \rightarrow L T$ is a labelling function, such that for each $e=(a, d o) \in E_{d a}$ there is a lt-labeled edge from the annotation a to the generic digital object do:

$$
\mathrm{l}_{d a}(a, d o)=\left\{\begin{array}{l}
\text { Annotate if } \exists \alpha \in \mathcal{A}_{a} \mid \alpha=\left(\mathrm{sn}, \text { Annotate, } \mathrm{st}_{\mathrm{sm}}, \mathrm{sm}, \mathrm{h}^{-1}(\text { do })\right) \\
\text { RelateTo if } \exists \alpha \in \mathcal{A}_{a} \mid \alpha=\left(\mathrm{sn}, \text { RelateTo, st } t_{\mathrm{sm}}, \mathrm{sm}, \mathrm{h}^{-1}(d o)\right)
\end{array}\right.
$$

The document-annotation hypertext is constructed by putting an edge between an annotation vertex and a digital object vertex, if the annotation is annotating or relating to that digital object. Note that we used $\mathrm{h}^{-1}(d o)$ in $E_{d a}$ to track the digital object back to its handle; the edge is then labeled with the corresponding link type. Each edge $e=(a, d o) \in E_{d a}$ always starts from an annotation $a \in A$, while $e \in E_{d a}$ which starts from a document $d \in D$ does not exist.

Figure 3 shows an example of document-annotation hypertext $H_{d a}$. In the figure, continuous lines, labeled with "A", indicate "annotate" links, while dotted lines, labeled with "R", indicate "relate to" links; annotations are labeled with the letter "a", while documents are labeled with the letter "d".

- $D=\left\{d_{1}, d_{2}, d_{3}, d_{4}, d_{5}\right\}$, we can assume that the subscript of each document indicates the time in which the document became an element of the set $D$;

- $A=\left\{a_{1}, a_{2}, a_{3}, a_{4}, a_{5}, a_{6}, a_{7}, a_{8}, a_{9}, a_{10}, a_{11}, a_{12}, a_{13}, a_{14}\right\}$, we can assume that the subscript of each annotation indicates the time in which the annotation became an element of the set $A$;

- we can express, for example:

- annotation sets concerning a document: $\left\{a_{1}, a_{2}\right\}$ is an annotation set concerning the document $d_{1}$;

- annotation sets concerning an annotation: $\left\{a_{8}, a_{9}\right\}$ is an annotation set concerning the annotation $a_{7}$;

- annotation threads concerning a document: $\left\{a_{1}, a_{3}, a_{4}\right\}$ is an annotation thread concerning the document $d_{1}$;

- annotation threads concerning an annotation: $\left\{a_{8}, a_{10}\right\}$ is an annotation thread concerning the annotation $a_{7}$;

- multiple annotation threads concerning a document: $\left\{a_{7}, a_{8}, a_{10}\right\}$ and $\left\{a_{12}, a_{13}, a_{14}\right\}$ are two different annotations threads both concerning the document $d_{3}$;

- multiple annotation threads concerning an annotation: $\left\{a_{8}, a_{10}\right\}$ and $\left\{a_{9}, a_{11}\right\}$ are two annotation threads both concerning the annotation $a_{7}$;

- nested annotation threads concerning a document: $\left\{a_{8}, a_{10}\right\}$ and $\left\{a_{9}, a_{11}\right\}$ are two different and nested annotation threads both concerning the document $d_{3}$. 


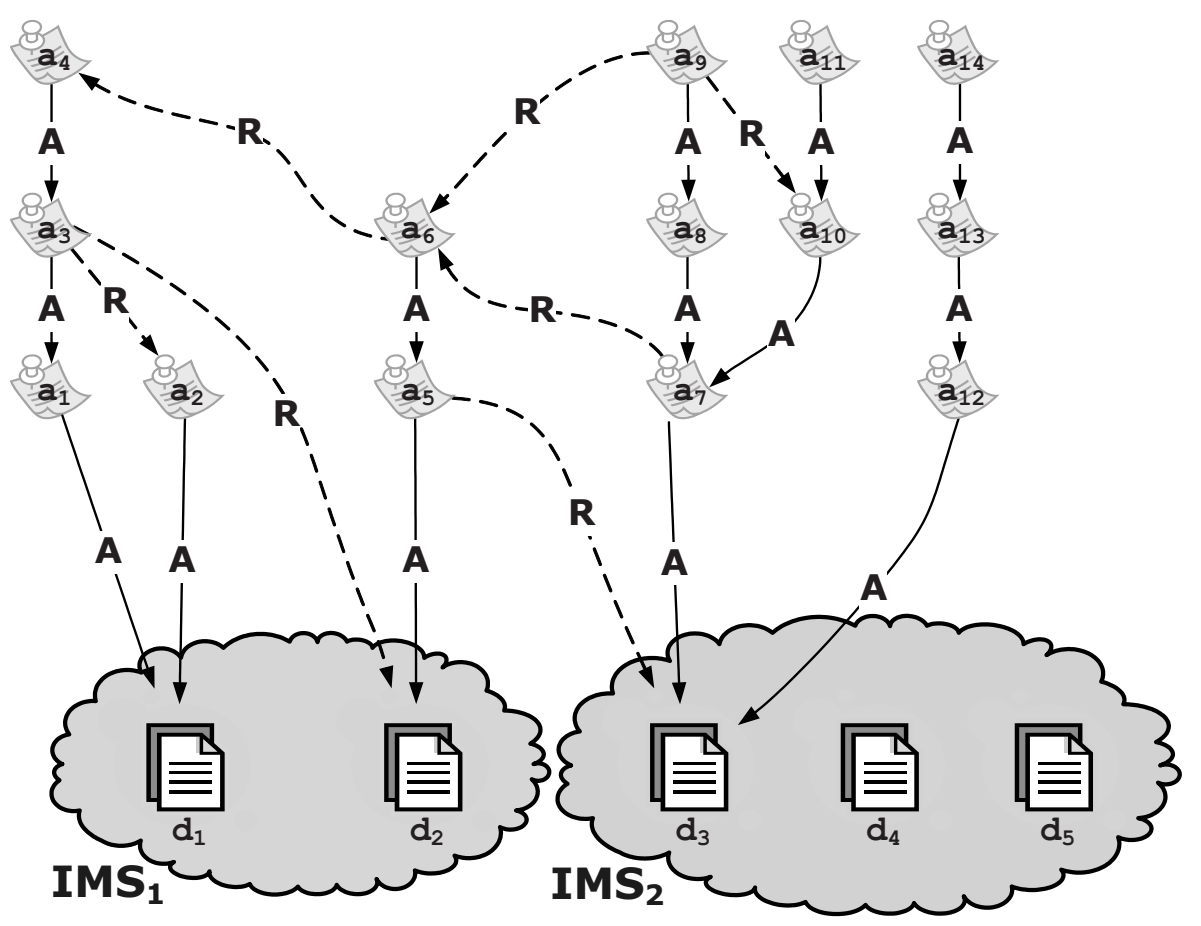

Fig. 3: Example of document-annotation hypertext $H_{d a}$

Figure 3 also points out another important feature of the documentannotation hypertext. It can span and cross the boundaries of the single IMS, as discussed in Sect. 3 . The $\mathrm{IMS}_{1}$ manages $d_{1}$ and $d_{2}$, while the $\mathrm{IMS}_{2}$ manages $d_{3}, d_{4}$, and $d_{5}$. There are annotations that act as a bridge between two IMSs: for example, $a_{5}$ annotates $d_{2}$, which is managed by $\mathrm{IMS}_{1}$, and refers to $d_{3}$, which is managed by $\mathrm{IMS}_{2}$.

\section{Conclusions}

We have presented different issues which concern the annotation of digital contents and we have shown how differently this seemingly simple and intuitive concept is perceived by users and designers, who often vary their definition of annotation according to the task at hand. In order to give an idea of how fragmentary the picture about annotations is, we have presented the different and coexisting perspectives on annotations with which we deal today.

Therefore, we have proposed a formal model of annotations on digital content, which not only captures all the aspects described above, but also effectively formalizes the time dimension entailed by annotations and explicitly introduces the notion of document-annotation hypertext. 
Finally, we have thoroughly discussed different application areas of the proposed formal model, which range from qualitative and formal comparison of existing system, definition of more specialized models derived from it, and design of advanced search algorithms which exploit annotations, to the development of flexible annotation services which can be easily integrated into existing IMSs in order to add annotation functionalities to them.

\section{References}

1. Abiteboul, S., Agrawal, R., Bernstein, P., Carey, M., Ceri, S., Croft, B., DeWitt, D., Franklin, M., Garcia-Molina, H., Gawlick, D., Gray, J., Haas, L., Halevy, A., Hellerstein, J., Ioannidis, Y., Kersten, M., Pazzani, M., Lesk, M., Maier, D., Naughton, J., Schek, H.J., Sellis, T., Silberschatz, A., Stonebraker, M., Snodgrass, R., Ullman, J.D., Weikum, G., Widom, J., Zdonik, S.: The Lowell Database Research Self-Assessment. Communications of the ACM (CACM) 48(5), 111-118 (2005)

2. Agosti, M., Albrechtsen, H., Ferro, N., Frommholz, I., Hansen, P., Orio, N., Panizzi, E., Pejtersen, A.M., Thiel, U.: DiLAS: a Digital Library Annotation Service. In: J.F. Boujut (ed.) Proc. International Workshop on Annotation for Collaboration - Methods, Tools, and Practices (IWAC 2005), pp. 91-101. CNRS - Programme société de l'information (2005)

3. Agosti, M., Benfante, L., Orio, N.: IPSA: A Digital Archive of Herbals to Support Scientific Research. In: T.M.T. Sembok, H.B. Zaman, H. Chen, S.R. Urs, S.H. Myaeng (eds.) Proc. 6th International Conference on Asian Digital Libraries - Digital Libraries: Technology and Management of Indigenous Knowledge (ICADL 2003), pp. 253-264. Lecture Notes in Computer Science (LNCS) 2911, Springer, Heidelberg, Germany (2003)

4. Agosti, M., Berretti, S., Brettlecker, G., del Bimbo, A., Ferro, N., Fuhr, N., Keim, D., Klas, C.P., Lidy, T., Norrie, M., Ranaldi, P., Rauber, A., Schek, H.J., Schreck, T., Schuldt, H., Signer, B., Springmann, M.: DelosDLMS - the Integrated DELOS Digital Library Management System. In: C. Thanos, F. Borri (eds.) DELOS Conference 2007 Working Notes, pp. 71-80. ISTI-CNR, Gruppo ALI, Pisa, Italy (2007)

5. Agosti, M., Bonfiglio-Dosio, G., Ferro, N.: A Historical and Contemporary Study on Annotations to Derive Key Features for Systems Design. International Journal on Digital Libraries http://dx.doi.org/10.1007/s00799-007-0010-0 (2007)

6. Agosti, M., Colotti, R., Gradenigo, G.: A Two-Level Hypertext Retrieval Model for Legal Data. In: E.A. Fox (ed.) Proc. 14th Annual International ACM SIGIR Conference on Research and Development in Information Retrieval (SIGIR 1991), pp. 316-325. ACM Press, New York, USA (1991)

7. Agosti, M., Coppotelli, T., Ferro, N., Pretto, L.: Exploiting Relevance Assessment for the Creation of an Experimental Test Collection to Evaluate Systems that Use Annotations. In: C. Thanos, F. Borri (eds.) DELOS Conference 2007 Working Notes, pp. 195-202. ISTI-CNR, Gruppo ALI, Pisa, Italy (2007)

8. Agosti, M., Ferro, N.: Annotations: Enriching a Digital Library. In: T. Koch, I.T. Sølvberg (eds.) Proc. 7th European Conference on Research and Advanced 
Technology for Digital Libraries (ECDL 2003), pp. 88-100. Lecture Notes in Computer Science (LNCS) 2769, Springer, Heidelberg, Germany (2003)

9. Agosti, M., Ferro, N.: An Information Service Architecture for Annotations. In: M. Agosti, H.J. Schek, C. Türker (eds.) Digital Library Architectures: Peerto-Peer, Grid, and Service-Orientation, Pre-proceedings of the 6th Thematic Workshop of the EU Network of Excellence DELOS, pp. 115-126. Edizioni Libreria Progetto, Padova, Italy (2004)

10. Agosti, M., Ferro, N.: A System Architecture as a Support to a Flexible Annotation Service. In: C. Türker, M. Agosti, H.J. Schek (eds.) Peer-to-Peer, Grid, and Service-Orientation in Digital Library Architectures: 6th Thematic Workshop of the EU Network of Excellence DELOS. Revised Selected Papers, pp. 147166. Lecture Notes in Computer Science (LNCS) 3664, Springer, Heidelberg, Germany (2005)

11. Agosti, M., Ferro, N.: Annotations as Context for Searching Documents. In: F. Crestani, I. Ruthven (eds.) Proc. 5th International Conference on Conceptions of Library and Information Science - Context: nature, impact and role, pp. 155-170. Lecture Notes in Computer Science (LNCS) 3507, Springer, Heidelberg, Germany (2005)

12. Agosti, M., Ferro, N.: Search Strategies for Finding Annotations and Annotated Documents: the FAST Service. In: H. Legind Larsen, G. Pasi, D. Ortiz-Arroyo, T. Andreasen, H. Christiansen (eds.) Proc. 7th International Conference on Flexible Query Answering Systems (FQAS 2006), pp. 270-281. Lecture Notes in Artificial Intelligence (LNAI) 4027, Springer, Heidelberg, Germany (2006)

13. Agosti, M., Ferro, N.: A Formal Model of Annotations of Digital Content. ACM Transactions on Information Systems (TOIS) 26(1), 1-55 (2008 (to appear))

14. Agosti, M., Ferro, N., Frommholz, I., Panizzi, E., Putz, W., Thiel, U.: Integration of the DiLAS Annotation Service into Digital Library Infrastructures. In: A. Blandford, J. Gow (eds.) Proc. 1st International Workshop on Digital Libraries in the Context of Users Broader Activities (DL-CUBA 2006), pp. 1-4. http://www.uclic.ucl.ac.uk/events/dl-cuba2006/ [last visited 2007, March 23] (2006)

15. Agosti, M., Ferro, N., Frommholz, I., Thiel, U.: Annotations in Digital Libraries and Collaboratories - Facets, Models and Usage. In: R. Heery, L. Lyon (eds.) Proc. 8th European Conference on Research and Advanced Technology for Digital Libraries (ECDL 2004), pp. 244-255. Lecture Notes in Computer Science (LNCS) 3232, Springer, Heidelberg, Germany (2004)

16. Agosti, M., Ferro, N., Orio, N.: Annotating Illuminated Manuscripts: an Effective Tool for Research and Education. In: M. Marlino, T. Sumner, F. Shipman (eds.) Proc. 5th ACM/IEEE-CS Joint Conference on Digital Libraries (JCDL 2005), pp. 121-130. ACM Press, New York, USA (2005)

17. Agosti, M., Ferro, N., Orio, N.: Graph-based Automatic Suggestion of Relationships among Images of Illuminated Manuscripts. In: H.M. Haddad, K.M. Liebrock, R. Chbeir, M.J. Palakal, S. Ossowski, K. Yetongnoon, R.L. Wainwright, C. Nicolle (eds.) Proc. 21st ACM Symposium on Applied Computing (SAC 2006), pp. 1063-1067. ACM Press, New York, USA (2006)

18. Agosti, M., Ferro, N., Orio, N.: Annotations as a Tool for Disclosing Hidden Relationships between Illuminated Manuscripts. In: Proc. 10th Congress of the Italian Association for Artificial Intelligence. Artificial Intelligence and HumanOriented Computing (AI*IA 2007). Lecture Notes in Computer Science (LNCS), Springer, Heidelberg, Germany (2007) 
19. Agosti, M., Ferro, N., Panizzi, E., Trinchese, R.: Annotation as a Support to User Interaction for Content Enhancement in Digital Libraries. In: A. Celentano, P. Mussio (eds.) Proc. Working Conference on Advanced Visual Interfaces (AVI 2006), pp. 151-154. ACM Press, New York, USA (2006)

20. Agosti, M., Smeaton, A. (eds.): Information Retrieval and Hypertext. Kluwer Academic Publishers, Norwell (MA), USA (1996)

21. Berners-Lee, T.: Universal Resource Identifiers in WWW. RFC 1630 (1994)

22. Berners-Lee, T., Fielding, R., Irvine, U.C., Masinter, L.: Uniform Resource Identifiers (URI): Generic Syntax. RFC 2396 (1998)

23. Bhagwat, D., Chiticariu, L., Tan, W.C., Vijayvargiya, G.: An Annotation Management System for Relational Databases. In: M.A. Nascimento, M.T. Özsu, D. Kossmann, R.J. Miller, J.A. Blakeley, K.B. Schiefer (eds.) Proc. 30th International Conference on Very Large Data Bases (VLDB 2004), pp. 900-911. Morgan Kaufmann (2004)

24. Bottoni, P., Civica, R., Levialdi, S., Orso, L., Panizzi, E., Trinchese, R.: MADCOW: a Multimedia Digital Annotation System. In: M.F. Costabile (ed.) Proc. Working Conference on Advanced Visual Interfaces (AVI 2004), pp. 55-62. ACM Press, New York, USA (2004)

25. Bottoni, P., Levialdi, S., Rizzo, P.: An Analysis and Case Study of Digital Annotation. In: N. Bianchi-Berthouze (ed.) Proc. 3rd International Workshop on Databases in Networked Information Systems (DNIS 2003), pp. 216-230. Lecture Notes in Computer Science (LNCS) 2822, Springer, Heidelberg, Germany (2003)

26. Buneman, P., Khanna, S., Tajima, K., Tan, W.C.: Archiving Scientific Data. ACM Transactions on Database Systems (TODS) 29(1), 2-42 (2004)

27. Buneman, P., Khanna, S., Tan, W.C.: Why and Where: A Characterization of Data Provenance. In: J. Van den Bussche, V. Vianu (eds.) Proc. 8th International Conference on Database Theory (ICDT 2001), pp. 316-330. Lecture Notes in Computer Science (LNCS) 1973, Springer, Heidelberg, Germany (2001)

28. Buneman, P., Khanna, S., Tan, W.C.: On Propagation of Deletions and Annotations Through Views. In: S. Abiteboul, P.G. Kolaitis, L. Popa (eds.) Proc. 21st ACM SIGMOD-SIGACT-SIGART Symposium on Principles of Database Systems (PODS 2002), pp. 150-158. ACM Press, New York, USA (2002)

29. Canova, M.G.: La tradizione europea degli erbari miniati e la scuola veneta. In: Di sana pianta. Erbari e taccuini di sanità: le radici storiche della nuova farmacologia, pp. 21-28. Panini, Modena, Italy (1988)

30. Cleverdon, C.W.: The Cranfield Tests on Index Languages Devices. In: K. Spärck Jones, P. Willett (eds.) Readings in Information Retrieval, pp. 47-60. Morgan Kaufmann Publisher, Inc., San Francisco, California, USA (1997)

31. Constantopoulos, P., Doerr, M., Theodoridou, M., Tzobanakis, M.: On Information Organization in Annotation Systems. In: G. Grieser, Y. Tanaka (eds.) Proc. International Workshop on Intuitive Human Interfaces for Organizing and Accessing Intellectual Assets, pp. 189-200. Lecture Notes in Computer Science (LNCS) 3359, Springer, Heidelberg, Germany (2004)

32. Davis, J.R., Huttenlocher, D.P.: Shared Annotation for Cooperative Learning. In: J.L. Schnase, E.L. Cunnius (eds.) Proc. 1st International Conference on Computer Support for Collaborative Learning (CSCL 1995), pp. 84-88. Lawrence Erlbaum Associates Inc., Mahwah (NJ), USA (1995) 
33. Ferro, N.: Formal Model and Conceptual Architecture of the Annotation Service for Dynamic Ubiquitous Knowledge Environments. PhD thesis, Department of Information Engineering. University of Padua, Italy (2004)

34. Frommholz, I., Brocks, H., Thiel, U., Neuhold, E., Iannone, L., Semeraro, G., Berardi, M., Ceci, M.: Document-Centered Collaboration for Scholars in the Humanities - The COLLATE System. In: T. Koch, I.T. Sølvberg (eds.) Proc. 7th European Conference on Research and Advanced Technology for Digital Libraries (ECDL 2003), pp. 434-445. Lecture Notes in Computer Science (LNCS) 2769, Springer, Heidelberg, Germany (2003)

35. Frommholz, I., Fuhr, N.: Probabilistic, Object-oriented Logics for Annotationbased Retrieval in Digital Libraries. In: G. Marchionini, M.L. Nelson, C.C. Marshall (eds.) Proc. 6th ACM/IEEE-CS Joint Conference on Digital Libraries (JCDL 2006), pp. 55-64. ACM Press, New York, USA (2006)

36. Giovè Marchioli, N.: Gli strumenti del sapere. I manoscritti padovani tra tipizzazioni generali e peculiarità locali. In: F. Piovan, L. Sitran Rea (eds.) Studenti, Università, città nella storia padovana. Atti del convegno (Padova, 6-8 febbraio 1998), pp. 47-71. Centro per la storia dell'Università di Padova. Contributi, 35, Lint, Trieste, Italia (2001)

37. Golder, S., Huberman, B.A.: Usage Patterns of Collaborative Tagging Systems. Journal of Information Science 32(2), 198-208 (2006)

38. Golovchinsky, G., Price, M.N., Schilit, B.N.: From Reading to Retrieval: Freeform Ink Annotations as Queries. In: F. Gey, M. Hearst, R. Tong (eds.) Proc. 22nd Annual International ACM SIGIR Conference on Research and Development in Information Retrieval (SIGIR 1999), pp. 19-25. ACM Press, New York, USA (1999)

39. Gonçalves, M.A., Fox, E.A., Watson, L.T., Kipp, N.A.: Streams, Structures, Spaces, Scenarios, Societies (5S): A Formal Model for Digital Libraries. ACM Transactions on Information Systems (TOIS) 22(2), 270-312 (2004)

40. Groth, D.P., Streefkerk, K.: Provenance and Annotation for Visual Exploration Systems. IEEE Transactions On Visualization And Computer Graphics 12(6), 1500-1510 (2006)

41. Halasz, F.G.: Reflections on NoteCards: Seven Issues for the Next Generation of Hypermedia Systems. Communications of the ACM (CACM) 31(7), 836-852 (1988)

42. Halasz, F.G., Moran, T.P., Trigg, R.H.: Notecards in a Nutshell. In: J.M. Carroll, P.P. Tanner (eds.) Proc. Conference on Human Factors in Computing Systems and Graphics Interface (CHI 1987), pp. 45-52. ACM Press, New York, USA (1987)

43. Handschuh, S., Staab, S. (eds.): Annotation for the Semantic Web. IOS Press, Amsterdam, The Netherlands (2003)

44. Hanks, P. (ed.): Collins Dictionary of the English Language. William Collins Sons \& Co. Ltd., Glasgow, UK (1979)

45. Hull, D.: Using Statistical Testing in the Evaluation of Retrieval Experiments. In: R. Korfhage, E. Rasmussen, P. Willett (eds.) Proc. 16th Annual International ACM SIGIR Conference on Research and Development in Information Retrieval (SIGIR 1993), pp. 329-338. ACM Press, New York, USA (1993)

46. Hwang, W.Y., Wang, C.Y., Sharples, M.: A study of multimedia annotation of Web-based materials. Computers \& Education 48(4), 680-699 (2007) 
47. IEI: Enciclopedia Italiana di scienze, lettere ed arti, Vol. XVII (GIAP-GS). Istituto della Enciclopedia Italiana, Istituto Poligrafico dello Stato, Roma, Italia (1951)

48. IEI: Vocabolario della lingua italiana, Vol. II (D-L). Istituto della Enciclopedia Italiana, Arti Grafiche Ricordi, Monotipia Olivieri, Milano, Italia (1987)

49. Ioannidis, Y., Maier, D., Abiteboul, S., Buneman, P., Davidson, S., Fox, E.A., Halevy, A., Knoblock, C., Rabitti, F., Schek, H.J., Weikum, G.: Digital library information-technology infrastructures. International Journal on Digital Libraries 5(4), 266-274 (2005)

50. Kahan, J., Koivunen, M.R.: Annotea: an open RDF infrastructure for shared Web annotations. In: V.Y. Shen, N. Saito, M.R. Lyu, M.E. Zurko (eds.) Proc. 10th International Conference on World Wide Web (WWW 2001), pp. 623-632. ACM Press, New York, USA (2001)

51. Marshall, C.C.: Annotation: from Paper Books to the Digital Library. In: R.B. Allen, E. Rasmussen (eds.) Proc. 2nd ACM International Conference on Digital Libraries (DL 1997), pp. 233-240. ACM Press, New York, USA (1997)

52. Marshall, C.C.: Toward an Ecology of Hypertext Annotation. In: R. Akscyn (ed.) Proc. 9th ACM Conference on Hypertext and Hypermedia (HT 1998): links, objects, time and space-structure in hypermedia systems, pp. 40-49. ACM Press, New York, USA (1998)

53. Marshall, C.C., Brush, A.J.B.: From Personal to Shared Annotations. In: L. Terveen, D. Wixon (eds.) Proc. Conference on Human Factors and Computing Systems (CHI 2002) - Extended Abstracts on Human Factors in Computer Systems, pp. 812-813. ACM Press, New York, USA (2002)

54. Marshall, C.C., Brush, A.J.B.: Exploring the Relationship between Personal and Public Annotations. In: H. Chen, H. Wactlar, C.C. Chen, E.P. Lim, M. Christel (eds.) Proc. 4th ACM/IEEE-CS Joint Conference on Digital Libraries (JCDL 2004), pp. 349-357. ACM Press, New York, USA (2004)

55. Nagao, K.: Digital Content Annotation and Transcoding. Artech House, Norwood (MA), USA (2003)

56. Navarro, G., Baeza-Yates, R.: Proximal Nodes: A Model to Query Document Databases by Content and Structure. ACM Transactions on Information Systems (TOIS) 15(4), 400-435 (1997)

57. NISO: ANSI/NISO Z39.88 - 2004 - The OpenURL Framework for ContextSensitive Services. National Information Standards Organization (NISO). http://www.niso.org/standards/standard_detail.cfm?std_id=783 [last visited 2007, March 23] (2005)

58. Ovsiannikov, I., Arbib, M.A., Mcneill, T.H.: Annotation Technology. International Journal of Human-Computer Studies 50, 329-362 (1999)

59. Paskin, N. (ed.): The DOI Handbook - Edition 4.4.1. International DOI Foundation (IDF). http://dx.doi.org/10.1000/186 [last visited 2007, March 23] (2006)

60. Phelps, T.A., Wilensky, R.: Multivalent Annotations. In: C. Peters, C. Thanos (eds.) Proc. 1st European Conference on Research and Advanced Technology for Digital Libraries (ECDL 1997), pp. 287-303. Lecture Notes in Computer Science (LNCS) 1324, Springer, Heidelberg, Germany (1997)

61. van Rijsbergen, C.J.: Information Retrieval, 2nd edn. Butterworths, London, England (1979)

62. Rocci, L.: Vocabolario greco italiano, 34 edizione. Società Editrice Dante Alighieri, Italia (1989) 
63. Salatiele: Ars notariae. In: G. Orlandelli (ed.) Opere dei maestri, Vol. 2. Giuffré, Milano, Italia (1961)

64. Salton, G., McGill, M.J.: Introduction to Modern Information Retrieval. McGraw-Hill, New York, USA (1983)

65. Shipman, F., Price, M.N., Marshall, C.C., Golovchinsky, G.: Identifying Useful Passages in Documents based on Annotation Patterns. In: T. Koch, I.T. Sølvberg (eds.) Proc. 7th European Conference on Research and Advanced Technology for Digital Libraries (ECDL 2003), pp. 101-112. Lecture Notes in Computer Science (LNCS) 2769, Springer, Heidelberg, Germany (2003)

66. Stein, L.D., Eddy, S., Dowell, R.: Distributed Sequence Annotation System (DAS) - Version 1.53. http://www . biodas.org/documents/spec.html [last visited 2007, March 23] (2002)

67. Thiel, U., Brocks, H., Frommholz, I., Dirsch-Weigand, A., Keiper, J., Stein, A., Neuhold, E.J.: COLLATE - A collaboratory supporting research on historic European films. International Journal on Digital Libraries 4(1), 8-12 (2004)

68. W3C: HTML 4.01 Specification - W3C Recommendation 24 December 1999. http://www.w3.org/TR/html4/ [last visited 2007, March 23] (1999)

69. W3C: Annotea Project. http://www.w3.org/2001/Annotea/ [last visited 2007, March 23] (2005)

70. W3C: Semantic Web . http://www.w3.org/2001/sw/ [last visited 2007, March 23] (2007)

71. Xi, W., Zhang, B., Chen, Z., Lu, Y., Yan, S., Ma, W.Y., Fox, E.A.: Link Fusion: A Unified Link Analysis Framework for Multi-Type Interrelated Data Objects. In: S. Feldman, M. Uretsky, M. Najork, C. Wills (eds.) Proc. 13th International Conference on World Wide Web (WWW 2004), pp. 319-327. ACM Press, New York, USA (2004) 